\title{
On the Norms of $r$-Toeplitz Matrices Involving Fibonacci and Lucas Numbers
}

\author{
Hasan Gökbaş ${ }^{1}$, Ramazan Türkmen²* \\ ${ }^{1}$ Semsi Tebrizi Anatolian Religious Vocational High School, Konya, Turkey \\ ${ }^{2}$ Science Faculty, Selcuk University, Konya, Turkey \\ Email: hgokbas@hotmail.com, 'rturkmen@selcuk.edu.tr.
}

Received 14 December 2015; accepted 30 May 2016; published 2 June 2016

Copyright $\odot 2016$ by authors and Scientific Research Publishing Inc.

This work is licensed under the Creative Commons Attribution International License (CC BY).

http://creativecommons.org/licenses/by/4.0/

(c) (i) Open Access

\section{Abstract}

Let us define $A=T_{r}\left[a_{i j}\right]$ to be a $n \times n \quad r$-Toeplitz matrix. The entries in the first row of $A=T_{r}\left[a_{i j}\right]$ are $a_{i j}=F_{i-j}$ or $a_{i j}=L_{i-j}$ where $F_{n}$ and $L_{n}$ denote the usual Fibonacci and Lucas numbers, respectively. We obtained some bounds for the spectral norm of these matrices.

\section{Keywords}

\section{$r$-Toeplitz Matrix, Fibonacci Numbers, Lucas Numbers, Spectral Norm}

\section{Introduction}

Toeplitz matrices arise in many different theoretical and applicative fields, in the mathematical modeling of all the problems where some sort of shift invariance occurs in terms of space or of time. As in computation of spline functions, time series analysis, signal and image processing, queueing theory, polynomial and power series computations and many other areas, typical problems modelled by Toeplitz matrices are the numerical solution of certain differential and integral equations [1]-[5].

Lots of article have been written so far, which concern estimates for spectral norms of Toeplitz matrices, which have connections with signal and image processing, time series analysis and many other problems [6]-[8]. Akbulak and Bozkurt found lower and upper bounds for the spectral norms of Toeplitz matrices with classical Fibonacci and Lucas numbers entries in [9]. Shen gave upper and lower bounds for the spectral norms of Toeplitz matrices with $k$-Fibonacci and $k$-Lucas numbers entries in [10].

In this paper, we derive expressions of spectral norms for $r$-Toeplitz matrices. We explain some preliminaries and well-known results. We thicken the identities of estimations for spectral norms of $r$-Toeplitz matrices.

*Corresponding author. 


\section{Preliminaries}

The Fibonacci and Lucas sequences $F_{n}$ and $L_{n}$ are defined by the recurrence relations

$$
F_{0}=0, F_{1}=1, F_{n}=F_{n-1}+F_{n-2} \text { for } n \geq 2
$$

and

$$
L_{0}=2, L_{1}=1, L_{n}=L_{n-1}+L_{n-2} \text { for } n \geq 2 .
$$

The rule can be used to extend the sequence backwards. Hence

$$
F_{-n}=(-1)^{n+1} F_{n}
$$

and

$$
L_{-n}=(-1)^{n} L_{n}
$$

If start from $n=0$, then the Fibonacci and Lucas sequence are given by

$\begin{array}{ccccccccc}n & 0 & 1 & 2 & 3 & 4 & 5 & 6 & 7 \\ F_{n} & 0 & 1 & 1 & 2 & 3 & 5 & 8 & 13 \\ L_{n} & 2 & 1 & 3 & 4 & 7 & 11 & 18 & 29 \\ F_{-n} & 0 & 1 & -1 & 2 & -3 & 5 & -8 & 13 \\ L_{-n} & 2 & -1 & 3 & -4 & 7 & -11 & 18 & -29\end{array}$

The following sum formulas the Fibonacci and Lucas numbers are well known [11] [12]:

$$
\begin{gathered}
\sum_{i=1}^{n-1} F_{i}^{2}=F_{n} F_{n-1} \\
\sum_{i=1}^{n-1} L_{i}^{2}=L_{n} L_{n-1}-2 \\
\sum_{k=1}^{n} F_{k} F_{k-1}= \begin{cases}F_{n}^{2}-1 & n \text { odd } \\
F_{n}^{2} & n \text { even }\end{cases} \\
\sum_{k=1}^{n} L_{k} L_{k-1}= \begin{cases}L_{n}^{2}+1 & n \text { odd } \\
L_{n}^{2}-4 & n \text { even }\end{cases}
\end{gathered}
$$

A matrix $T_{r}=\left[t_{i j}\right] \in M_{n, n}(\mathbb{C})$ is called a $r$-Toeplitz matrix if it is of the form

$$
t_{i j}= \begin{cases}t_{i-j}, & i \leq j \\ r t_{i-j}, & i>j\end{cases}
$$

Obviously, the $r$-Toeplitz matrix $T$ is determined by parameter $r$ and its first row elements $t_{0}, t_{-1}, \cdots t_{1-n}$, thus we denote $T=T_{r}\left(t_{0}, t_{-1}, \cdots, t_{1-n}\right)$. Especially, let $r=1$, the matrix $T$ is called a Toeplitz matrix.

A matrix $S T_{r}=\left[t_{i j}\right] \in M_{n, n}(\mathbb{C})$ is called a symmetric $r$-Toeplitz matrix if it is of the form

$$
t_{i j}= \begin{cases}r t_{i-j}, & i<j \\ t_{i-j}, & i \geq j\end{cases}
$$

Obviously, the symmetric $r$-Toeplitz matrix $T$ is determined by parameter $r$ and its last row elements $t_{n-1}, t_{n-2}, \cdots, t_{0}$, thus we denote $T=S T_{r}\left(t_{n-1}, t_{n-2}, \cdots, t_{0}\right)$. Especially, let $r=1$, the matrix $T$ is called a Toeplitz matrix.

The Euclidean norm of the matrix $A$ is defined as

$$
\|A\|_{E}=\left(\sum_{i, j=1}^{n}\left|a_{i j}\right|^{2}\right)^{1 / 2}
$$


The singular values of the matrix $A$ is

$$
\sigma_{i}=\sqrt{\lambda_{i}\left(A^{*} A\right)}
$$

where $\lambda_{i}$ is an eigenvalue of $A^{*} A$ and $A^{*}$ is conjugate transpose of matrix $A$. For a square matrix $A$, the square roots of the maximum eigenvalues of $A^{*} A$ are called the spectral norm of $A$. The spectral norm of the matrix $A$ is

$$
\|A\|_{2}=\max \left(\sigma_{i}\right)
$$

The following inequality holds,

$$
\frac{1}{\sqrt{n}}\|A\|_{E} \leq\|A\|_{2} \leq\|A\|_{E} .
$$

Define the maximum column lenght norm $c_{1}$, and the maximum row lenght norm $r_{1}$ of any matrix $A$ by

$$
r_{1}(A)=\max _{i} \sqrt{\sum_{j}\left|a_{i j}\right|^{2}}
$$

and

$$
c_{1}(A)=\max _{j} \sqrt{\sum_{i}\left|a_{i j}\right|^{2}}
$$

respectively. Let $A, B$ and $C$ be $m \times n$ matrices. If $A=B \circ C$ then

$$
\|A\|_{2} \leq r_{1}(B) c_{1}(C)[13] .
$$

Theorem 1 [9]. Let $A=T\left[a_{i j}\right]$ be a Toeplitz matrix satisfying $a_{i j}=F_{i-j}$, then

$$
\begin{cases}\sqrt{\frac{2}{n}\left(F_{n}^{2}\right)} \leq\|A\|_{2} \leq \sqrt{\left(1+F_{n} F_{n-1}\right)\left(F_{n} F_{n-1}\right)} & n \text { even } \\ \sqrt{\frac{2}{n}\left(F_{n}^{2}-1\right)} \leq\|A\|_{2} \leq \sqrt{\left(1+F_{n} F_{n-1}\right)\left(F_{n} F_{n-1}\right)} & n \text { odd }\end{cases}
$$

where \|\|$_{2}$ is the spectral norm and $F_{n}$ denotes the $n$th Fibonacci number.

Theorem 2 [9]. Let $A=T\left[a_{i j}\right]$ be a Toeplitz matrix satisfying $a_{i j}=L_{i-j}$, then

$$
\begin{cases}\sqrt{\frac{2}{n}\left(L_{n}^{2}-4\right)} \leq\|A\|_{2} \leq \sqrt{\left(L_{n} L_{n-1}-1\right)\left(L_{n} L_{n-1}+2\right)} & n \text { even } \\ \sqrt{\frac{2}{n}\left(L_{n}^{2}+1\right)} \leq\|A\|_{2} \leq \sqrt{\left(L_{n} L_{n-1}-1\right)\left(L_{n} L_{n-1}+2\right)} & n \text { odd }\end{cases}
$$

where \|\|$_{2}$ is the spectral norm and $L_{n}$ denotes the $n$th Lucas number.

\section{Result and Discussion}

Theorem 3. Let $A=T_{r}\left[a_{i j}\right]$ be a r-Toeplitz matrix satisfying $a_{i j}=F_{i-j}$, where $r \in \mathbb{C}$.

- $|r| \geq 1, \begin{cases}\sqrt{\frac{2}{n}\left(F_{n}^{2}-1\right)} \leq\|A\|_{2} \leq|r| \sqrt{(n-1) F_{n} F_{n-1}} & n \text { odd } \\ \sqrt{\frac{2}{n}\left(F_{n}^{2}\right)} \leq\|A\|_{2} \leq|r| \sqrt{(n-1) F_{n} F_{n-1}} & n \text { even }\end{cases}$

- $|r|<1, \begin{cases}\sqrt{\frac{2}{n}|r|^{2}\left(F_{n}^{2}-1\right)} \leq\|A\|_{2} \leq \sqrt{(n-1) F_{n} F_{n-1}} & n \text { odd } \\ \sqrt{\frac{2}{n}|r|^{2}\left(F_{n}^{2}\right)} \leq\|A\|_{2} \leq \sqrt{(n-1) F_{n} F_{n-1}} & n \text { even }\end{cases}$ 
where $\|.\|_{2}$ is the spectral norm and $F_{n}$ denotes the $n$th Fibonacci number.

Proof. The matrix $A$ is of the form

$$
A=\left[\begin{array}{ccccc}
F_{0} & F_{-1} & \cdots & F_{2-n} & F_{1-n} \\
r F_{1} & F_{0} & \cdots & F_{3-n} & F_{2-n} \\
\vdots & \vdots & \ddots & \vdots & \vdots \\
r F_{n-2} & r F_{n-3} & \cdots & F_{0} & F_{-1} \\
r F_{n-1} & r F_{n-2} & \cdots & r F_{1} & F_{0}
\end{array}\right]
$$

Then we have,

$$
\|A\|_{F}^{2}=n F_{0}^{2}+\sum_{i=1}^{n-1}|r|^{2}(n-i) F_{i}^{2}+\sum_{i=1}^{n-1}(n-i) F_{-i}^{2}
$$

hence, when $|r| \geq 1$ we obtain

$$
\|A\|_{F}^{2} \geq n F_{0}^{2}+2 \sum_{i=1}^{n-1}(n-i) F_{i}^{2}=n F_{0}^{2}+2 \sum_{i=1}^{n-1} \sum_{k=1}^{i} F_{k}^{2}=2 \sum_{k=1}^{n} F_{k} F_{k-1}
$$

that is

$$
\|A\|_{2} \geq \begin{cases}\sqrt{\frac{2}{n}\left(F_{n}^{2}-1\right)} & n \text { odd } \\ \sqrt{\frac{2}{n}\left(F_{n}^{2}\right)} & n \text { even }\end{cases}
$$

On the other hand, let the matrices $B$ and $C$ as

$$
B=\left[\begin{array}{ccccc}
F_{0} & 1 & \cdots & 1 & 1 \\
r & F_{0} & \cdots & 1 & 1 \\
\vdots & \vdots & \ddots & \vdots & \vdots \\
r & r & \cdots & F_{0} & 1 \\
r & r & \cdots & r & F_{0}
\end{array}\right]
$$

and

$$
C=\left[\begin{array}{ccccc}
F_{0} & F_{-1} & \cdots & F_{2-n} & F_{1-n} \\
F_{1} & F_{0} & \cdots & F_{3-n} & F_{2-n} \\
\vdots & \vdots & \ddots & \vdots & \vdots \\
F_{n-2} & F_{n-3} & \cdots & F_{0} & F_{-1} \\
F_{n-1} & F_{n-2} & \cdots & F_{1} & F_{0}
\end{array}\right]
$$

such that $A=B \circ C$. Then

$$
r_{1}(B)=\max _{i} \sqrt{\sum_{j}\left|b_{i j}\right|^{2}}=\sqrt{\sum_{j=0}^{n-1}\left|b_{n j}\right|^{2}}=\sqrt{|r|^{2}(n-1)}=|r| \sqrt{n-1}
$$

and

$$
c_{1}(C)=\max _{j} \sqrt{\sum_{i}\left|c_{i j}\right|^{2}}=\sqrt{\sum_{i=0}^{n-1}\left|c_{i n}\right|^{2}}=\sqrt{\sum_{i=0}^{n-1} F_{i}^{2}}=\sqrt{F_{n} F_{n-1}} .
$$

We have

$$
\|A\|_{2} \leq|r| \sqrt{(n-1) F_{n} F_{n-1}}
$$

when $|r|<1$ we also obtain

$$
\|A\|_{F}^{2} \geq n F_{0}^{2}+2|r|^{2} \sum_{i=1}^{n-1}(n-i) F_{i}^{2}=n F_{0}^{2}+2|r|^{2} \sum_{i=1}^{n-1} \sum_{k=1}^{i} F_{k}^{2}=2|r|^{2} \sum_{k=1}^{n} F_{k} F_{k-1}
$$


that is

$$
\|A\|_{2} \geq \begin{cases}\sqrt{\frac{2}{n}|r|^{2}\left(F_{n}^{2}-1\right)} & n \text { odd } \\ \sqrt{\frac{2}{n}|r|^{2}\left(F_{n}^{2}\right)} & n \text { even }\end{cases}
$$

On the other hand, let the matrices $B$ and $C$ as

$$
B=\left[\begin{array}{ccccc}
F_{0} & 1 & \cdots & 1 & 1 \\
r & F_{0} & \cdots & 1 & 1 \\
\vdots & \vdots & \ddots & \vdots & \vdots \\
r & r & \cdots & F_{0} & 1 \\
r & r & \cdots & r & F_{0}
\end{array}\right]
$$

and

$$
C=\left[\begin{array}{ccccc}
F_{0} & F_{-1} & \cdots & F_{2-n} & F_{1-n} \\
F_{1} & F_{0} & \cdots & F_{3-n} & F_{2-n} \\
\vdots & \vdots & \ddots & \vdots & \vdots \\
F_{n-2} & F_{n-3} & \cdots & F_{0} & F_{-1} \\
F_{n-1} & F_{n-2} & \cdots & F_{1} & F_{0}
\end{array}\right]
$$

such that $A=B \circ C$. Then

$$
r_{1}(B)=\max _{i} \sqrt{\sum_{j}\left|b_{i j}\right|^{2}}=\sqrt{\sum_{j=0}^{n-1}\left|b_{n j}\right|^{2}}=\sqrt{n-1}
$$

and

$$
c_{1}(C)=\max _{j} \sqrt{\sum_{i}\left|c_{i j}\right|^{2}}=\sqrt{\sum_{i=0}^{n-1}\left|c_{i n}\right|^{2}}=\sqrt{\sum_{i=0}^{n-1} F_{i}^{2}}=\sqrt{F_{n} F_{n-1}} .
$$

We have

$$
\|A\|_{2} \leq \sqrt{(n-1) F_{n} F_{n-1}} .
$$

Thus, the proof is completed.

Corollary 4. Let $A=S T_{r}\left(F_{n-1}, F_{n-2}, \cdots, F_{0}\right)$ be a symmetric $r$-Toeplitz matrix, where $r$, then

- $|r| \geq 1, \begin{cases}\sqrt{\frac{2}{n}\left(F_{n}^{2}-1\right)} \leq\|A\|_{2} \leq|r| \sqrt{(n-1) F_{n} F_{n-1}} & n \text { odd } \\ \sqrt{\frac{2}{n}\left(F_{n}^{2}\right)} \leq\|A\|_{2} \leq|r| \sqrt{(n-1) F_{n} F_{n-1}} & n \text { even }\end{cases}$

- $|r|<1, \begin{cases}\sqrt{\frac{2}{n}|r|^{2}\left(F_{n}^{2}-1\right)} \leq\|A\|_{2} \leq \sqrt{(n-1) F_{n} F_{n-1}} & n \text { odd } \\ \sqrt{\frac{2}{n}|r|^{2}\left(F_{n}^{2}\right)} \leq\|A\|_{2} \leq \sqrt{(n-1) F_{n} F_{n-1}} & n \text { even }\end{cases}$

where $\|\cdot\|_{2}$ is the spectral norm and $F_{n}$ denotes the $n$th Fibonacci number.

Proof. Owing to the fact that the sum of all elements squares are equal in matrices (1) and (2), the proof is concluded analogously in the proof of previous theorem.

Theorem 5. Let $A=T_{r}\left[a_{i j}\right]$ be a r-Toeplitz matrix satisfying $a_{i j}=L_{i-j}$, where $r \in \mathbb{C}$. 
- $|r| \geq 1, \begin{cases}\sqrt{\frac{2\left(L_{n}^{2}+1\right)}{n}} \leq\|A\|_{2} \leq \sqrt{\left[|r|^{2}(n-1)+1\right]\left[L_{n} L_{n-1}+2\right]} & n \text { odd } \\ \sqrt{\frac{2\left(L_{n}^{2}-4\right)}{n}} \leq\|A\|_{2} \leq \sqrt{\left[|r|^{2}(n-1)+1\right]\left[L_{n} L_{n-1}+2\right]} & n \text { even }\end{cases}$

- $|r|<1, \begin{cases}\sqrt{\frac{2|r|^{2}\left(L_{n}^{2}+1\right)+4 n\left(1-|r|^{2}\right)}{n}} \leq\|A\|_{2} \leq \sqrt{n\left(L_{n} L_{n-1}+2\right)} \quad n \text { odd } \\ \sqrt{\frac{2|r|^{2}\left(L_{n}^{2}-4\right)+4 n\left(1-|r|^{2}\right)}{n}} \leq\|A\|_{2} \leq \sqrt{n\left(L_{n} L_{n-1}+2\right)} \quad n \text { even }\end{cases}$

where $\|.\|_{2}$ is the spectral norm and $L_{n}$ denotes the $n$th Lucas number.

Proof. The matrix $A$ is of the form

$$
A=\left[\begin{array}{ccccc}
L_{0} & L_{-1} & \cdots & L_{2-n} & L_{1-n} \\
r L_{1} & L_{0} & \cdots & L_{3-n} & L_{2-n} \\
\vdots & \vdots & \ddots & \vdots & \vdots \\
r L_{n-2} & r L_{n-3} & \cdots & L_{0} & L_{-1} \\
r L_{n-1} & r L_{n-2} & \cdots & r L_{1} & L_{0}
\end{array}\right]
$$

then we have

$$
\|A\|_{F}^{2}=n L_{0}^{2}+\sum_{i=1}^{n-1}|r|^{2}(n-i) L_{i}^{2}+\sum_{i=1}^{n-1}(n-i) L_{-i}^{2}
$$

hence when $|r| \geq 1$ we obtain

$$
\|A\|_{2}^{2} \geq n L_{0}^{2}+2 \sum_{i=1}^{n-1}(n-i) L_{i}^{2}=n L_{0}^{2}+2 \sum_{i=1}^{n-1} \sum_{k=1}^{i} L_{k}^{2}=n L_{0}^{2}+2 \sum_{k=1}^{n} L_{k} L_{k-1}
$$

that is

$$
\|A\|_{2} \geq \begin{cases}\sqrt{\frac{2\left(L_{n}^{2}+1\right)}{n}} & n \text { odd } \\ \sqrt{\frac{2\left(L_{n}^{2}-4\right)}{n}} & n \text { even }\end{cases}
$$

On the other hand let matrices $B$ and $C$ be as

$$
B=\left[\begin{array}{ccccc}
1 & 1 & \cdots & 1 & 1 \\
r & 1 & \cdots & 1 & 1 \\
\vdots & \vdots & \ddots & \vdots & \vdots \\
r & r & \cdots & 1 & 1 \\
r & r & \cdots & r & 1
\end{array}\right]
$$

and

$$
C=\left[\begin{array}{ccccc}
L_{0} & L_{-1} & \cdots & L_{2-n} & L_{1-n} \\
L_{1} & L_{0} & \cdots & L_{3-n} & L_{2-n} \\
\vdots & \vdots & \ddots & \vdots & \vdots \\
L_{n-2} & L_{n-3} & \cdots & L_{0} & L_{-1} \\
L_{n-1} & L_{n-2} & \cdots & L_{1} & L_{0}
\end{array}\right]
$$


such that $A=B \circ C$. Then

$$
r_{1}(B)=\max _{i} \sqrt{\sum_{j}\left|b_{i j}\right|^{2}}=\sqrt{\sum_{j=0}^{n-1}\left|b_{n j}\right|^{2}}=\sqrt{|r|^{2}(n-1)}=|r| \sqrt{n-1}
$$

and

$$
c_{1}(C)=\max _{j} \sqrt{\sum_{i}\left|c_{i j}\right|^{2}}=\sqrt{\sum_{i=0}^{n-1}\left|c_{i n}\right|^{2}}=\sqrt{\sum_{i=0}^{n-1} L_{i}^{2}}=\sqrt{F_{n} F_{n-1}+2} .
$$

We have

$$
\|A\|_{2} \leq \sqrt{\left[|r|^{2}(n-1)+1\right]\left[L_{n} L_{n-1}+2\right]}
$$

when $|r|<1$ we also obtain

$$
\|A\|_{2}^{2} \geq n L_{0}^{2}+2|r|^{2} \sum_{i=1}^{n-1}(n-i) L_{i}^{2}=n L_{0}^{2}+2|r|^{2} \sum_{i=1}^{n-1} \sum_{k=1}^{i} L_{k}^{2}=4 n+2|r|^{2} \sum_{k=1}^{n} L_{k} L_{k-1}
$$

that is

$$
\|A\|_{2} \geq \begin{cases}\sqrt{\frac{2|r|^{2}\left(L_{n}^{2}+1\right)+4 n\left(1-|r|^{2}\right)}{n}} & n \text { odd } \\ \sqrt{\frac{2|r|^{2}\left(L_{n}^{2}-4\right)+4 n\left(1-|r|^{2}\right)}{n}} & n \text { even }\end{cases}
$$

On the other hand, let matrices $B$ and $C$ be as

$$
B=\left[\begin{array}{ccccc}
1 & 1 & \cdots & 1 & 1 \\
r & 1 & \cdots & 1 & 1 \\
\vdots & \vdots & \ddots & \vdots & \vdots \\
r & r & \cdots & 1 & 1 \\
r & r & \cdots & r & 1
\end{array}\right]
$$

and

$$
C=\left[\begin{array}{ccccc}
L_{0} & L_{-1} & \cdots & L_{2-n} & L_{1-n} \\
L_{1} & L_{0} & \cdots & L_{3-n} & L_{2-n} \\
\vdots & \vdots & \ddots & \vdots & \vdots \\
L_{n-2} & L_{n-3} & \cdots & L_{0} & L_{-1} \\
L_{n-1} & L_{n-2} & \cdots & L_{1} & L_{0}
\end{array}\right]
$$

such that $A=B \circ C$. Then

$$
r_{1}(B)=\max _{i} \sqrt{\sum_{j}\left|b_{i j}\right|^{2}}=\sqrt{\sum_{j=0}^{n-1}\left|b_{n j}\right|^{2}}=\sqrt{n}
$$

and

$$
c_{1}(C)=\max _{j} \sqrt{\sum_{i}\left|c_{i j}\right|^{2}}=\sqrt{\sum_{i=0}^{n-1}\left|c_{i n}\right|^{2}}=\sqrt{\sum_{i=0}^{n-1} L_{i}^{2}}=\sqrt{F_{n} F_{n-1}+2} .
$$

We have

$$
\|A\|_{2} \leq \sqrt{n\left(L_{n} L_{n-1}+2\right)}
$$


Thus, the proof is completed.

Corollary 6. Let $A=S T_{r}\left(L_{n-1}, L_{n-2}, \cdots, L_{0}\right)$ be a symmetric $r$-Toeplitz matrix, where $r \in \mathbb{C}$, then

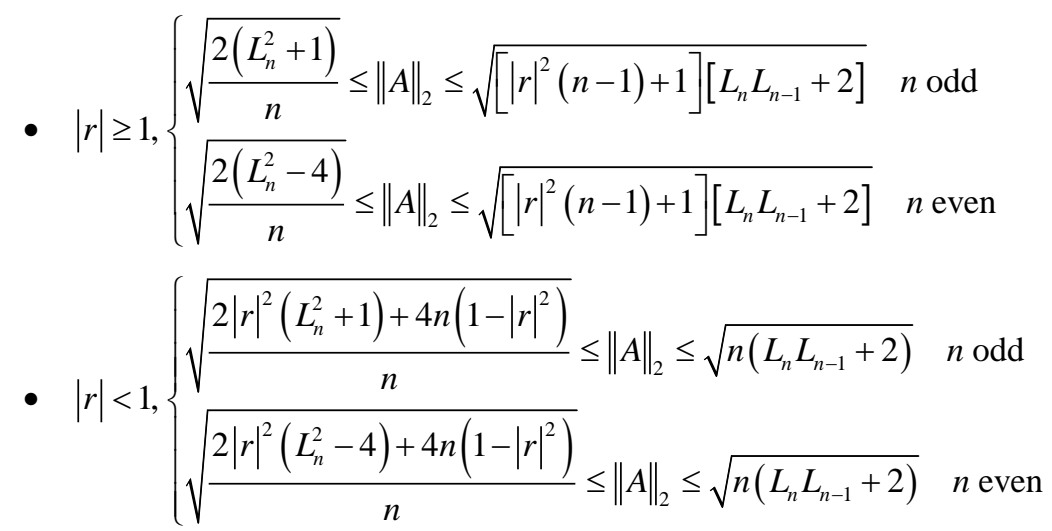

where \|\|$_{2}$ is the spectral norm and $L_{n}$ denotes the $n$th Lucas number.

Proof. Owing to the fact that the sum of all elements squares are equal in matrices (1) and (2), the proof is concluded analogously in the proof of previous theorem.

\section{Numarical Examples}

Example 7. Let $A=T_{r}\left(F_{0}, F_{-1}, \cdots, F_{1-n}\right)$ be a r-Toeplitz matrix, in which $F_{i}(i=0,1, \cdots, n-1)$ denotes the Fibonacci number, where $r \in \mathbb{C}$. From Table 1, it is easy to find that upper bounds for the spectral norm, of Theorem 3 are more sharper than Theorem 1 (see Table 1).

\begin{tabular}{ccc} 
Table 1. Numerical results of $a_{i j}=F_{i-j}, r=1$. & \\
\hline $\boldsymbol{n}$ & Theorem 1 & Theorem 3 \\
\hline 2 & $\sqrt{2}$ & $\sqrt{1}$ \\
3 & $\sqrt{6}$ & $\sqrt{4}$ \\
4 & $\sqrt{42}$ & $\sqrt{18}$ \\
5 & $\sqrt{240}$ & $\sqrt{60}$ \\
6 & $\sqrt{1640}$ & $\sqrt{200}$ \\
$\ldots$ & $\cdots$ & $\cdots$ \\
$n$ & $\sqrt{\left(1+F_{n} F_{n-1}\right)\left(F_{n} F_{n-1}\right)}$ & $\sqrt{(n-1) F_{n} F_{n-1}}$ \\
\hline
\end{tabular}

Table 2. Numerical results of $a_{i j}=L_{i-j}, \quad r=1$.

\begin{tabular}{ccc}
\hline $\boldsymbol{n}$ & Theorem 2 & Theorem 5 \\
\hline 2 & $\sqrt{10}$ & $\sqrt{10}$ \\
3 & $\sqrt{154}$ & $\sqrt{42}$ \\
4 & $\sqrt{810}$ & $\sqrt{120}$ \\
5 & $\sqrt{6004}$ & $\sqrt{395}$ \\
6 & $\sqrt{39400}$ & $\sqrt{1200}$ \\
$\ldots$ & $\ldots$ & $\cdots$ \\
$n$ & $\sqrt{\left(L_{n} L_{n-1}-1\right)\left(L_{n} L_{n-1}+2\right)}$ & $\sqrt{(n-1)\left(L_{n} L_{n-1}+2\right)}$ \\
\hline
\end{tabular}


Example 8. Let $A=T_{r}\left(L_{0}, L_{-1}, \cdots, L_{1-n}\right)$ be a r-Toeplitz matrix, in which $L_{i}(i=0,1, \cdots, n-1)$ denotes the Lucas number, where $r \in \mathbb{C}$. From Table 2, it is easy to find that upper bounds for the spectral norm, of Theorem 5 are more sharper than Theorem 2, when $n \geq 2$ (see Table 2).

\section{References}

[1] Dubbs, A. and Edelman, A. (2014) Infinite Random Matrix Theory. Tridiagonal Bordered Toeplitz Matrices and the Moment Problem. arXiv:1502.04931v1.

[2] Erbas, C. and Tanik, M.M. (1995) Generating Solutions to the N-Queens Problems Using 2-Circulants. Mathematics Magazine, 68, 343-356. http://dx.doi.org/10.2307/2690923

[3] Ngondiep, E., Serra-Capizzano, S. and Sesana, D. (2010) Spectral Features and Asymptotic Proporties for g-Circulant and g-Toeplitz Sequence. SIAM Journal on Matrix Analysis and Applications, 31, 1663-1687. http://dx.doi.org/10.1137/090760209

[4] Szegö, G. (1958) Toeplitz Forms and Their Applications. University of California Press.

[5] Hudson, R.E., Reed, C.W., Chen, D. and Lorenzelli, F. (1998) Blind Beamforming on a Randomly Distributed sensor Array System. Journal on Selected Areas in Communications, 16, 1555-1567.

[6] Gray, R.M. and Davisson, L.D. (2005) An Introduction to Statistical Signal Processing. Cambridge University Press, London.

[7] Wei, Y., Cai, J. and Ng, M.K. (2004) Computing Moore-Penrose Inverses of Toeplitz Matrices by Newton's Iteration. Mathematical and Computer Modelling, 40, 181-191. http://dx.doi.org/10.1016/j.mcm.2003.09.036

[8] Chou, W.S., Du, B.S. and Shiue, P.J.-S. (2008) A Note on Circulant Transition Matrices in Markov Chains. Linear Algebra and Its Applications, 429, 1699-1704. http://dx.doi.org/10.1016/j.laa.2008.05.004

[9] Akbulak, M. and Bozkurt, D. (2008) On the Norms of Toeplitz Matrices Involving Fibonacci and Lucas Numbers. Hacettepe Journal of Mathematics and Statistics, 37, 89-95.

[10] Shen, S. (2012) On the Norms of Toeplitz Matrices Involving k-Fibonacci and k-Lucas Numbers. International Journal of Contemporary Mathematical Sciences, 7, 363-368.

[11] Vajda, S. (1989) Fibonacci and Lucas Numbers and the Golden Section: Theory and Applications. Ellis Horwood Ltd.

[12] Koshy, T. (2001) Fibonacci and Lucas Numbers with Applications. A Wiley-Interscience Publication.

[13] Horn, R.A. and Johnson, C.R. (1991) Topics in Matrix Analysis. Cambridge University Press, Cambridge. http://dx.doi.org/10.1017/CBO9780511840371 